\title{
Breve reseña sobre el Río Uruguay
}

\author{
Míguez, D. ${ }^{(1)}$ \\ Contacto: dmiguez@latu.org.uy \\ (1) Departamento Aguas y Productos Químicos - Laboratorio Tecnológico del Uruguay (LATU)
}

\begin{abstract}
Resumen
La cuenca del río Uruguay está compartida entre tres países del MERCOSUR. Si bien la calidad de sus aguas es en general buena, los principales problemas se centran en el riesgo de generar condiciones eutróficas en algunos de sus tramos, por altas concentraciones de nutrientes y que han dado lugar a eventos de floraciones algales.
\end{abstract}

\section{Abstract}

The river Uruguay watershed is a shared resource of three MERCOSUR countries. Even though its water quality is in general good, the main problem is the risk of generating eutrophic conditions in some areas because of high nutrient content and especially phosphorus concentrations, which have given rise to algal blooming.

\section{Introducción}

$\mathrm{L}$ a presente es una recopilación de información contenida en diversas fuentes bibliográficas y las conclusiones preliminares basadas en ensayos realizados por el Latu en muestras extraídas en una determinada zona del río

\section{Geografía}

El Río Uruguay es un curso de agua internacional cuya cuenca forma parte de los territorios de Argentina, Brasil y Uruguay en un área cercana a los $339.000 \mathrm{Km} 2$. Se origina en la Sierra do Mar (Brasil). Su longitud es de $1.800 \mathrm{Km}$, desembocando en el Río de la Plata. Su curso transcurre un $32 \%$ por territorio brasilero, un $38 \%$ forma límite entre Argentina y Brasil y un 30\% forma límite entre Argentina y Uruguay 1. La cuenca del Río Uruguay se encuentra entre las latitudes $28010^{\prime} \mathrm{S}$ y $37008^{\prime} \mathrm{S}^{8}$

\section{Régimen}

Funciona como un sistema hídrico compuesto por dos partes diferenciales: el cauce principal del Río por donde pasa el mayor volumen de agua, y las zonas costeras donde el agua tiene una menor renovación y se produce un estacionamiento de la misma.1 El régimen del Río Uruguay es uno de los mejores conocidos, ya

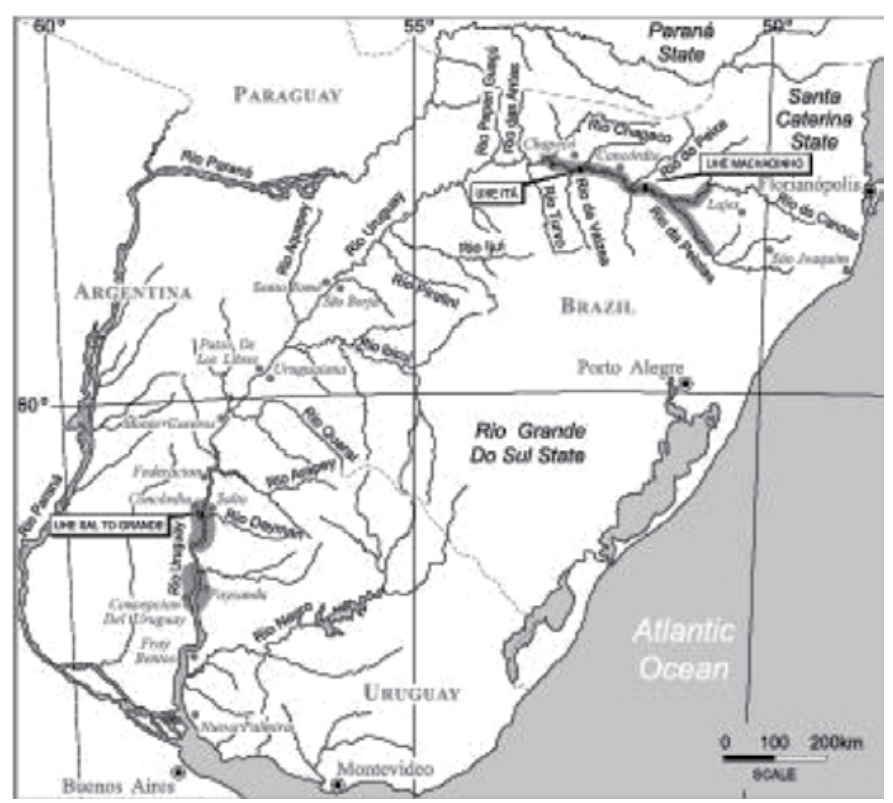

que se poseen observaciones hidrográficas desde el año 1892 , el mismo es muy irregular, consecuencia de la irregularidad de las lluvias que se descargan sobre su cuenca. En su origen, se descargan anualmente lluvias de $2000 \mathrm{~mm}$, y en su cuenca las mismas oscilan entre 1000 y $2000 \mathrm{~mm}$ anuales. ${ }^{8}$

\section{Caudales}

Los caudales en invierno y primavera, se mantienen por lo general con valores altos, con promedios del orden de los $7000 \mathrm{~m} 3 / \mathrm{s}$. Durante el verano se produce el estiaje, alcanzando caudales inferiores a los $2000 \mathrm{~m} 3 / \mathrm{s}$. El caudal medio es del orden de los $4500 \mathrm{~m} 3 / \mathrm{s}$.

El máximo caudal conocido hasta la fecha se produjo en el mes de abril de 1959, con 36000 m3/s, medido en el Puerto de Concordia. ${ }^{8}$

\section{Geología}

Por sus características generales, el Uruguay se incluye entre los ríos que drenan rocas sedimentarias marinas. ${ }^{2}$ Es el río más joven de la cuenca del Plata, formada también por el Paraguay y el Paraná. Su cuenca se asienta sobre rocas sedimentarias y volcánicas de la cuenca del Paraná. Las rocas ígneas (en forma de depósitos de lava) de las montañas de la Serra Geral, predominan y cubren las rocas sedimentarias del Mesozoico y Neo-Paleozoico. El suelo en general es arcilloso y poco profundo. ${ }^{8}$

\section{Regiones y represas}

Está conformado por una serie de rápidos. La represa hidroeléctrica de Itá comenzó a llenarse en diciembre de 1999. Las cascadas de Yucumã (o Moconá), en la desembocadura del río Peperi, representa una caída de $12 \mathrm{~m}$ en rápidos de $1800 \mathrm{~m}$ de largo, la más ancha de Sudamérica. En la desembocadura del río Quarai, del Salto Grande caía el agua desde una altura de 9 m en una extensión de $3 \mathrm{~km}$; estos rápidos fueron anegados en 1979 después de la construcción de la represa. La cascada Yucumã divide al Uruguay en medio y superior, mientras que Salto Grande es considerado el límite entre el medio e inferior.

\section{Afluentes y navegabilidad}

\section{Margen izquierda:}

Río UUHY (Brasil) desemboca unos pocos $\mathrm{km}$. al norte de Barra Concepción (Misiones - R.A.J. Al sur: Río IBICUY (Bra- 
sil), muy caudaloso, desemboca en el Uruguay, frente a Yapeyú (Corrientes).

Río CUAREIM: sirve de límite entre la R. O. del Uruguay y el Brasil y desemboca a $6 \mathrm{~km}$. al norte de Monte Caseros (Corrientes - Argentina).

Río ARAPEY: R. O. del Uruguay. Posee aguas termales muy reconocidas, desaguando en la Represa de Salto Grande, frente a Santa Ana (Entre Ríos).

Río DAYMAN (R.O.U.), con aguas termales, desemboca unos pocos $\mathrm{km}$ al sur de la ciudad de Salto (R.O.U.).

Río QUEGUAY (R.O.U.): desemboca unos km al norte de Paysandú (R.O.U.), frente a la isla homónima.

Río NEGRO (R.O.U.): el mayor tributario del Río Uruguay, nace en la frontera brasileña/ uruguaya, se desarrolla por el territorio de la R.O. del Uruguay recorriendo $550 \mathrm{~km}$. para finalmente

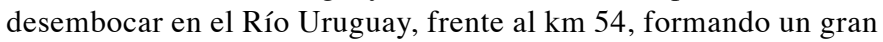
delta de $5 \mathrm{~km}$ de ancho.

\section{Margen derecha:}

Río CHAPELCO (Brasil). Luego recibe al Río PEPIRI GUAZU que es límite entre Brasil y Argentina.

Río AGUAPEY (Corrientes): nace cerca del límite entre Corrientes y Misiones, corre por territorio correntino a través de $290 \mathrm{~km}$. para desembocar finalmente unos $\mathrm{km}$ al sur de Alvear (Corrientes - R.A.).

Río MIRIÑAY: nace en la Laguna del Ibera (Corrí ente s-R. A.), tiene una longitud de $219 \mathrm{~km}$. y desemboca al norte de Monte Caseros (Corrientes-RA.).

Río MOCORETA: tiene una longitud de $143 \mathrm{~km}$. y sirve de límite entre las Provincias de Corrientes y Entre Ríos.

Río GUALEGUAYCHU (Entre Ríos-RA.): tiene una longitud de $120 \mathrm{~km}$. posee márgenes bajas y arboladas a $18 \mathrm{~km}$. antes de su desembocadura en el Uruguay se halla la ciudad de Gualeguaychú (R.A.), hasta aquí es navegable para embarcaciones de 9 pies de calado. $^{7}$

Las condiciones hidrológicas del Bajo Uruguay se ven influenciadas fuertemente por la represa hidroeléctrica de Salto Grande. Las variaciones históricas del nivel solían ser pequeñas, bajando solamente 1,2 metros durante las sequías. A pesar de ésto, actualmente las inundaciones pueden exceder 10 metros de altura. Los puentes hacia arriba del de Fray Bentos fueron por eso construidos en dos niveles, permitiendo operar en esas dos condiciones.

\section{Usos de la tierra}

En el Alto Uruguay la agricultura se basa en la soja, el maíz y los frijoles. En el Medio y Bajo Uruguay, prevalecen la cría intensiva de ganado y el cultivo de soja y arroz. Quedan solamente fragmentos de monte indígena. En la sección brasileña, la vegetación primaria y secundaria cubren cerca de $17.5 \%$ de la tierra. Las áreas reforestadas con pinos (Pinus elliottii), ocupan otro 3\%; el resto son pasturas.

La densidad de población es de 39 habitantes por kilómetro cuadrado, residiendo casi el $45 \%$ en áreas rurales.

\section{Biodiversidad y riqueza pesquera}

Las especies de peces del Alto Uruguay son parecidas a la del Bajo Paraná, con gran biodiversidad y productividad, para ser un clima subtropical.

Entre las ciudades de Colón $(236 \mathrm{~km})$ y Fray Bentos $(102 \mathrm{~km})$ muchas islas quiebran al Río Uruguay. En la desembocadura del río Gualeguaychú River, las islas desaparecen y el río se ensancha hasta 8 a $12 \mathrm{~km}$. Hay una serie de caneles que vinculan al Paraná y al Uruguay, influenciados en su velocidad y dirección por el Río de la Plata. En Nueva Palmira, el Río Uruguay desemboca en el estuario. Se han descrito más de 200 especies de peces en el Río
Uruguay. El pez más frecuente en el Bajo Uruguay es el curimbatá (Prochilodus lineatus), de origen marino. La voga (Schizodon nasutus) y el bagre armado (Pterodoras granulosus) le siguen en frecuencia. Otros son: la piava (Leporinus obtusidens), el dorado (Salminus maxillosus) y el Patí (Luciopimelodus pati).

\section{Calidad del agua}

Para controlar la calidad el agua se creó la Comisión Administradora del Río Uruguay (C.A.R.U.) por las Repúblicas Argentina y Oriental del Uruguay mediante el "Estatuto del Río Uruguay" el 26 de febrero de 1975. Tiene sede en Paysandú y cinco delegados de cada país. Participan la Dirección Nacional de Medio Ambiente (DINAMA), el Servicio de Oceanografía. Meteorología e Hidrografía de ia Armada (SOHMA) y el Servicio de Hidrografía Naval argentino. De sus campañas de monitoreos se desprende que, en promedio, es un río poco contaminado, pero se han constatado problemas ambientales provenientes principalmente de efectos locales, más bien costeros. En el cuerpo principal hay algunas zonas con alguna contaminación: Bella Unión-Monte Caseros, punto de entrada al sistema; Salto-Concordia, punto aguas abajo de la principal concentración urbana en el tramo; y la estación de Fray Bentos que coincide con la descarga del Río Gualeguaychú donde se ha encontrado algunos valores altos de metales pesados, sobre todo en plomo.

Cerca de las ciudades, la contaminación de los efluentes cloacales no tratados y en la cuenca superior, la de aguas residuales de granjas de cerdos y de aves, producen niveles altos localizados. El oxígeno disuelto, en condiciones normales, está cercano a los de saturación, el pH próximo a la neutralidad, mientras que la conductividad y la alcalinidad aumentan a lo largo del río, aunque aparecen anomalías en ciertas áreas.

\section{Impactos ambientales}

Los niveles de mercurio son nueve veces mayores que los aceptables en el Alto Uruguay y los ríos Canoas, Peixe y Pelotas. La fuente es probablemente las plantas de celulosas en estas cuencas.

Los pesticidas, herbicidas, nutrientes y metales pesados (cromo de curtiembres) provienen de actividades agronómicas e industriales. Además, la mayoría de los efluentes domésticos son vertidos sin tratamiento, lo que ha tenido muchas consecuencias, como por ejemplo la introducción del ambiente favorable para el cólera en la zona alta del río.

\section{Nutrientes y eutrofización}

En situaciones sin impactos antrópicos, la productividad fitoplanctónica baja del río se debe a una corriente intensa, concentración baja de nutrientes y alta turbiedad. La productividad de las comunidades macrofíticas es también bastante baja debido a la escasez de lagunas. La productividad primaria baja hace que las comunidades dependan en gran forma de la materia orgánica originada en la rivera.

La sobrecarga orgánica de algunas secciones también provoca eutrofización, dando por resultado floraciones de algas tóxicas. Hay sedimentos contaminados por cobre, níquel y zinc. El origen principal son probablemente los pesticidas y herbicidas y las plantas de galvanoplastia y metalurgia.

La distribución de fósforo inorgánico y nitrógeno inorgánico muestra en general pequeñas diferencias de concentración en el canal respecto de las costas. Según algunos estudios, los valores del nitrógeno y del fósforo son $0,653 \mathrm{mg} / 1$, en promedio, respectivamente. El valor del cociente de Redfield es 16 para agua y 
fitoplancton marinos. Este cociente para aguas del curso principal del Río Uruguay presenta variaciones que estarían indicando probables efectos debidos a escorrentía de áreas cultivadas por uso de fertilizantes en algunos casos y en el lago del embalse.

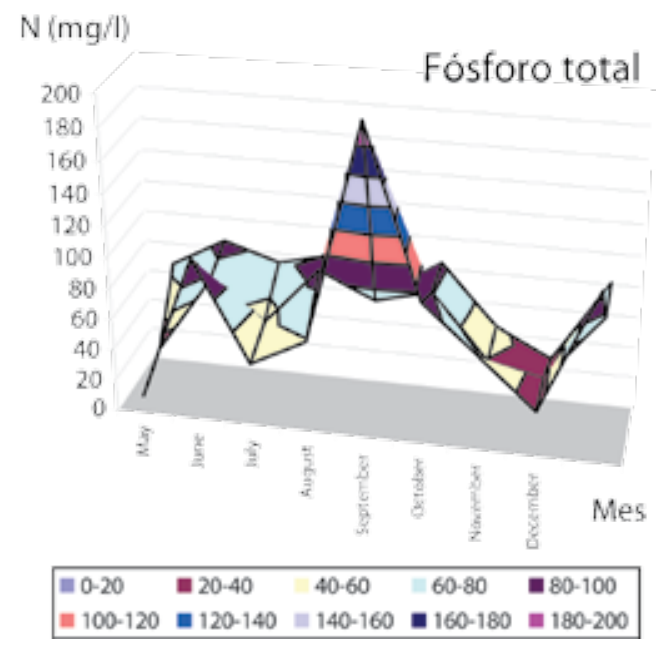

El embalsado de los ríos ha provocado cambios dramáticos en las características propias de los ríos. En relación a los parámetros biológicos, el embalsamamiento de un río altera marcadamente el componente planctónico. El promedio de la concentración de fósforo total en el agua varía entre $93 \mathrm{mg} / \mathrm{l}$ y $130 \mathrm{mg} / \mathrm{l}$ (valores máximos: $720 \mathrm{mg} / \mathrm{l}$ ), indicando eutrofización. El nitrógeno total sigue esta tendencia, con promedios entre 336 y $941 \mathrm{mg} / 1$ (máximo: $5,430 \mathrm{mg} / \mathrm{l})$. (8). La concentración media de nutrientes (NT=553 $\mu \mathrm{g} / \mathrm{l} ; \mathrm{NO} 3=394 \mu \mathrm{g} / \mathrm{l} ; \mathrm{NH} 4=13 \mu \mathrm{g} / \mathrm{l} ; \mathrm{PT}=66 \mu \mathrm{g} / 1$ y PO $4=23 \mu \mathrm{g} / \mathrm{l})$, puede ser considerada característica de ambientes mesotróficos a eutróficos. Por otro lado, tanto el valor de fósforo total $(67 \mu \mathrm{g} / \mathrm{l})$ como el de clorofila a $(4 \mu \mathrm{g} / \mathrm{l})$ clasifican a la zona de la represa como eutrófica.

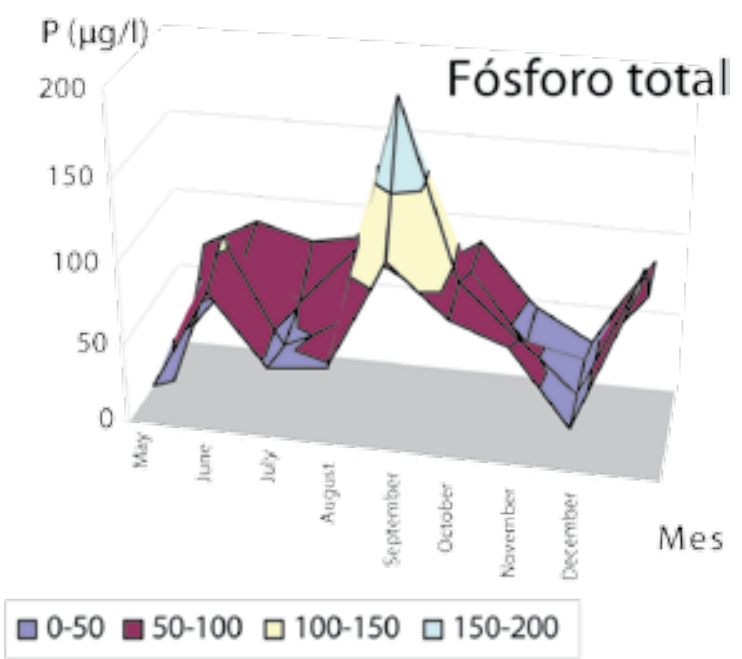

En estudios realizados por el Laboratorio Tecnológico del Uruguay se analizaron todos los parámetros de la normativa nacional y otros más exigentes (AOX, por ejemplo). No se observaron mayores problemas en ningún parámetro salvo en los nutrientes. Las tendencias en los valores de fósforo y de nitrógeno durante el año 2005 tuvieron un pico en el mes de setiembre, tal como se ve en las gráficas anteriores. El posterior descenso en las concentraciones de los nutrientes se vio acompañado de un crecimiento de algas desmedido, síntoma de la eutrofización. Asimismo, en la temporada estival se determinaron niveles por encima de los permitidos de microcistina-LR, toxina cancerígena, cuyo órgano blanco es el hígado y que es producida por las cianobacterias (un tipo de algas unicelulares). El pH aumentó a más de 9 en el evento de floración algal.

Las áreas de más alta contaminación son río abajo de las ci- udades y centros industriales tales como Salto-Concordia, Paysandú-Colón y la desembocadura del río Gualeguaychú. Metales pesados y agroquímicos no se encuentran en cantidades elevadas. Sin embargo se han hallado en especies acuáticas organoclorados en Paysandú. En un estudio fueron encontrados metabolitos del DDT en peces.

La falta de un sistema de pretratamiento en la red cloacal de las ciudades propicia los vertidos urbanos e industriales en forma cruda al Río Uruguay. Por ejemplo, concentraciones de Cromo 120 veces superior al límite máximo permitido por C.A.R.U.(Uso 4) en el Río Uruguay han sido encontradas en Paysandú.

Durante el muestreo de playas desarrollado por C.A.R.U. entre noviembre y diciembre de 2000 , se detectan en el balneario municipal, zona $\mathrm{B}$, concentraciones de coliformes fecales de hasta 800.000 y $550.000 \mathrm{UFC} / 100 \mathrm{ml}$, valores que superan varias miles de veces el límite máximo permitido por C.A.R.U. para zonas de Uso 2 (recreación con contacto directo).

El efluente industrial que se vierte directamente al río en esa zona, registraba en junio de 2000, valores de coliformes fecales de $65.000 \mathrm{UFC} / 1 \mathrm{OOml}$, siendo el límite fijado por DI.NA.MA. de 5.000 UFC/1 OOml. Dicho efluente representa un caudal de 4.000.000 litros/día.

Las ciudades carecen de plantas de tratamiento de los efluentes urbanos, por lo que los mismos acceden en forma cruda al Río Uruguay. La ausencia de Planes de Desarrollo Urbano incide en los problemas de contaminación que se detectan tanto en los efluentes industriales como en sus zonas costeras.

\section{Conclusiones}

$\mathrm{D}$ los estudios realizados en el Laboratorio Tecnológico del Uruguay y de la recopilación bibliográfica se desprende que los principales problemas de contaminación actual en algunas zonas del Río Uruguay se originan en una gestión ambiental que no ha sido planificada para proteger este curso de agua. Se requieren no solamente obras de infraestructura que realicen tratamientos a las aguas servidas de las ciudades ribereñas, sino también buenas prácticas de agricultura y el manejo integrado de la cuenca para poder gestionar en forma eficiente el recurso, sin contaminarlo. Los futuros aportes de nutrientes y de otros contaminantes de los emprendimientos industriales que se instalen en las costas de este curso de agua deberán ser tenidos en cuenta para no afectar la salud ambiental y la ecología.

\section{Referencias}

Siete años de estudios en calidad de aguas en el Río Uruguay. 1994. En: Publicaciones C.A.R.U. (Serie de divulgación, 2).

III Seminario sobre calidad de las aguas y contaminación: 29-30 de noviembre de 2001. Colón

Paradiso, M.,Chalar, G. Influencia de las condiciones ambientales de la represa de Salto Grande aguas abajo: resultados preliminares

Cardini, J. /et al/. Estudio y modelación de la calidad del agua del Río Uruguay : efectos de descargas cloacales e Industriales en el entorno de las ciudades de Concepción del Uruguay y Gualeguaychú. En: III Seminario sobre calidad de aguas y contaminación: 29-30 de noviembre de 2001. Paysandú

Appratto, M. Estudio de las principales descargas industriales al Río Uruguay y sus afluentes. En: III Seminario sobre calidad de aguas y contaminación : 29-30 de noviembre de 2001. Paysandú

Resultados analíticos de LATU sobre muestras de agua del Río Uruguay.

Primer seminario sobre la navegación en el Río Uruguay : 19-20 de noviembre de 1992. Paysandú

Zaniboni Filho, E; Schulz, U. Migratory fishes of the Uruguay River. IDRC 